Cahiers Charlevoix

Études franco-ontariennes
Cahiers Charlevoix Études franco-ontariennes

or Crevenerix of

\title{
Essai sur le français parlé en Ontario : entre représentations et légitimité
}

\section{Julie Boissonneault}

Volume 12, 2018

URI : https://id.erudit.org/iderudit/1048917ar

DOI : https://doi.org/10.7202/1048917ar

Aller au sommaire du numéro

\section{Éditeur(s)}

Société Charlevoix

Presses de l’Université d’Ottawa

\section{ISSN}

1203-4371 (imprimé)

2371-6878 (numérique)

Découvrir la revue

Citer cet article

Boissonneault, J. (2018). Essai sur le français parlé en Ontario : entre représentations et légitimité. Cahiers Charlevoix, 12, 89-116.

https://doi.org/10.7202/1048917ar
Résumé de l'article

Pour sa deuxième contribution, Julie Boissonneault, qui a précédemment analysé les contraintes institutionnelles qui entravent l'intention et la réalisation des études universitaires en français en Ontario, se penche ici sur la perception qu'on a de la langue des Franco-Ontariens. La langue française parlée en Ontario fait souvent l'objet d'un discours dépréciatif qui établit la compétence des Franco-Ontariens à l'aune de leurs manquements au français de référence. Or, ce discours, qui ne tient pas compte de l'enjeu de la variation interne de toute langue et des processus naturels d'évolution des parlers, nourrit des représentations à l'égard du parler qui sont souvent partielles ou fausses et qui, en retour, peuvent mener à un sentiment d'incompétence chez le locuteur franco-ontarien. Cet essai propose un regard critique sur la trialectique - "discours épilinguistique / représentation linguistique / sécurité-insécurité linguistique » - et met de l'avant la primauté de la description concertée, détaillée et critique de toute variété de langue dans son aménagement "formel ». 


\title{
Essai sur le français parlé en Ontario : entre représentations et légitimité
}

\author{
JuLIE BoIsSONNEAULT
}

Département d'études françaises

Université Laurentienne 


\section{SOMMAIRE}

$\begin{array}{ll}\text { INTRODUCTION } & 91\end{array}$

1. Des attitudes et des RePréSEntations linguistiQues 94

1.1. Entre le discours et les jugements : valorisation ou dévalorisation de la langue $\quad 94$

1.2. La variation linguistique : un phénomène universel 98

2. DU COMPLEXE À TENIR UN DISCOURS EN « BON 》 FRANÇAIS 101 2.1. La compétence linguistique : entre la sécurité et l'insécurité 101

2.2. Contrer l'insécurité linguistique pour légitimer le français au Québec et en Acadie $\quad 104$

2.3. Les Franco-Ontariens face à la langue 107

3. De la lÉGITIMATION ET DE LA RECONNAISSANCE DU FRANCO-ONTARIEN 109

3.1. La description du corpus de la langue $\quad 110$

3.2. La dénomination d'une langue : un acte délicat 


\title{
Essai sur le français parlé en Ontario : entre représentations et légitimité
}

\begin{abstract}
Si, en Ontario français, la pleine reconnaissance de la collectivité a toujours été une conquête imprécise et de longue haleine, c'est que le territoire du sujet minoritaire, lieu clair de son engagement dans son monde, porte les signes d'une subtile illégitimité, ressentie au jour le jour dans le vécu de chaque individu.
\end{abstract}

FRANÇOIS PARÉ ${ }^{1}$

\section{INTRODUCTION}

Paraissait dans la revue Liaison ${ }^{2}$, au printemps 2016, un article que signait Hélène Koscielniak, romancière franco-ontarienne ${ }^{3}$, sur le français parlé en Ontario ${ }^{4}$. Dans son texte, Koscielniak soulève le dilemme quant à la langue à utiliser pour faire parler ses personnages : doit-elle faire appel à la langue familière telle qu'elle se parle réellement dans le Nord ontarien afin de légitimer ses personnages ou doit-elle recourir à une langue normée et accessible à un plus grand lectorat de langue française ? Par ces questions - que posent d'autres auteurs, quels que soient leur lieu ou leur langue -, l'auteure met de l'avant des questions sur « l'acceptation de la langue orale des Franco-Ontariens ${ }^{5}$ ». Elle

1. François Paré, « Manifeste pour une mémoire occultée », Revue du NouvelOntario, no 41, 2016, p. 14.

2. Hélène Koscielniak, «Le Tarois : dossier », Liaison, La revue des arts, $\mathrm{n}^{\circ} 171$, printemps 2016, p. 6-19.

3. Originaire du Nord-Est ontarien, Hélène Koscielniak a signé cinq romans : Marraine (2007), Carnet de bord (2009), Contrepoids (2011), Filleul (2012) et Frédéric (2014) publiés aux éditions L'Interligne (Ottawa) et pour lesquels elle a obtenu le prix Littérature éclairée du Nord à quatre reprises (Association des auteures et auteurs de l'Ontario français, https://aaof.ca, consulté le 9 mars 2018).

4. Je tiens à remercier ma collègue Renée Corbeil qui a lu la première mouture de cet article et m'a fait part de conseils judicieux.

5. Communiqué, Liaison, $\mathrm{n}^{\circ}$ 171, le 22 mars 2016. 
aborde les questions épineuses de la variation en français parlé en Ontario et, ce faisant, de l'image que l'on s'en fait, image qui nourrit l'insécurité qu'éprouvent les locuteurs à l'endroit de leur parler. Pour contrer cette insécurité, elle propose de légitimer le parler franco-ontarien en le nommant, et ce, dans le but de rehausser sa valorisation par le sens d'appartenance. Elle a ainsi recours à l'appellation « tarois ${ }^{6} »$ pour distinguer le français ontarien du français de référence et des autres « français » parlés au Canada.

Je me rends compte que les gens d'ici parlent ce quej'ai décidé de nommer le tarois. C'est une langue bâtarde, oui. C'est une langue qui vient du français. C'est une soupe qui vient du canadienfrançais, du joual, de l'anglais et d'autres mots ramassés ici et là à d'autres langues, avec un accent spécifique ${ }^{7}$.

Les médias se sont empressés de donner suite aux propos tenus par Koscielniak ${ }^{8}$ en s'emparant de réactions diverses suscitées chez les uns et les autres. Parler de langue n'est, après tout, jamais chose neutre. Parler de langue, c'est à la fois parler d'un bien commun et d'une manifestation individuelle, celui de l'individu, en l'occurrence, ici, du Franco-Ontarien, qui s'exprime dans cette langue. C'est aussi parler de l'opinion qu'il se forge de sa langue ou de l'opinion qu'il croit que d'autres s'en font, de la valeur qu'elle revêt à ses yeux et de l'importance qu'il lui accorde. Ces attributs ne se donnent pas en vase clos, mais à l'aune d'attitudes et de représentations.

6. Le vocable « tarois » est la troncation du mot « ontarois », terme conçu dans les années 1980 pour désigner les Franco-Ontariens. Bien que certains intellectuels aient privilégié cette appellation, elle n'a pas été retenue dans l'usage qui lui a préféré la dénomination « franco-ontarien » (Yolande Grisé, " Ontarois, on l'est encore!", Ottawa, Le Nordir, 2002, 435 p.). Voir aussi Roger Bernard, De Québécois à Ontarois. La communauté franco-ontarienne, Hearst, Le Nordir, 1988, 185 p.

7. Propos tenus par Hélène Koscielniak à l'émission Grands Lacs café, « Le Tarois - La langue des Franco-Ontariens ? », animée par Éric Robitaille, RadioCanada, le samedi 26 mars 2016, http://ici.radio-canada.ca (consulté le 9 mars 2018).

8. Émission Couleurs locales, UnisTV, « Derrière nos barreaux » (S2, épisode 25) diffusée le 10 avril 2016, http://unis.ca/videos?v=ynigihh4tcqr3 (consulté le 9 mars 2018) ; «Le Tarois - La langue des Franco-Ontariens ? », Émission Grands Lacs café, op. cit. ; « Le Tarois... Le français des Franco-Ontariens », Émission 24/7 réalisée par Caroline Leal, TFo, 2015, http://www.tfo.org (consulté le 28 juin 2016) ; Bienvenu Senga, "Le Tarois et l'insécurité linguistique », Le Voyageur, le mercredi 11 mai 2016, p. 5. 
Deux grands constats sur la langue française et, de façon plus spécifique, sur l'usage qu'en font les Franco-Ontariens, se dégagent des propos tenus par Koscielniak : il y a, d'abord, le sentiment linguistique à s'exprimer en français, puis la valorisation - individuelle ou collective - de la variété de langue. Le premier découle de la qualité que les Franco-Ontariens attribuent à leur expression, jugement souvent porté par comparaison à une langue de référence et qui se manifeste dans les appellations dévalorisantes qui la qualifient ou les discours qu'ils tiennent à son égard. On entend ainsi couramment des propos qualifiant la langue de " slang » et de « franglais » ou indiquant qu'une personne estime « ne pas bien parler » ou « parler un mauvais français ». Le second - la valorisation - $s$ 'inscrit dans la reconnaissance sociale que l'on attribue à la langue française en Ontario, reconnaissance qui valorise ou dévalorise son usage, tout particulièrement sa spécificité linguistique. Cette reconnaissance ne se joue pas sur le seul tableau du statut que l'on accorde à la langue - sa légitimité sociopolitique et juridique ${ }^{9}-$, mais aussi sur sa légitimité symbolique, c'est-à-dire celle qu'on lui attribue de par la description que l'on en fait.

La perception de la compétence en une langue et de la légitimité symbolique de cette langue relève ainsi de la représentation que s'en font les locuteurs. Il s'agit ici des attitudes qu'entretiennent les locuteurs du français parlé en Ontario à son égard. Il y a donc interrelation en ce que la compétence ou l'incompétence perçue, qu'elle soit réelle ou non, est fonction de l'image que l'on se fait de ce que la langue est ou devrait être, des attitudes et des croyances qu'on en a, d'une part, et de l'appellation ou des vocables par lesquels on la désigne ou des propos par lesquels on la qualifie, d'autre part. C'est dire que la façon dont on parle

9. La langue française n'est pas reconnue comme langue co-officielle en Ontario. Seule la langue anglaise l'est. Elle est cependant protégée par la Loi de 1986 sur les services en français qui lui confère un statut dans l'administration publique de certaines régions. Elle est aussi reconnue comme langue d'enseignement (à tous les paliers scolaires) et langue de la justice. 
d'une langue trahit l'image que l'on s'en fait et l'attitude qu'on adopte à son égard tend à confirmer cette image.

C'est cette trialectique entre les discours, la représentation et la sécurité-insécurité linguistique qui m'intéresse. Je tenterai, dans cet essai, d'exposer comment les aléas des représentations et des attitudes linguistiques transmises sur le français parlé en Ontario agissent sur sa légitimité symbolique. Il s'agira donc de montrer comment, dans une perspective systémique interrelationnelle, les discours que l'on tient sur les langues émanent d'attitudes et de représentations et que ces discours, nourris à même la description que l'on fait du parler, sont les vecteurs par excellence de la sécurité ou de l'insécurité des locuteurs à utiliser une langue et à lui conférer une certaine légitimité. Sera aussi abordée l'importance de l'aménagement d'une langue par de grands travaux de description pour légitimer son usage et pour faire valoir sa spécificité.

\section{Des atTITUdes et DES REPRÉSENTATIONS LingUistiQUeS \\ 1.1. Entre le discours et les jugements : \\ valorisation ou dévalorisation de la langue}

Toute langue fait l'objet de représentations - qu'elles soient partagées ou non par les membres qui en composent la communauté linguistique - qui prennent forme dans les discours tenus à son sujet. Les représentations émanent de locuteurs individuels parce que chaque personne a accès à au moins une langue ${ }^{10}$ et que chaque personne se prononce sur la connaissance qu'elle a de sa langue, connaissance souvent considérée comme connaissance de la langue.

[L]e langage occupe dans la vie de chaque individu et de chaque communauté une telle place qu'il ne peut pas ne pas être lui-même objet de représentation sociale. [...] Tout locuteur prétend à une certaine compétence dans la connaissance des mécanismes linguistiques, ne serait-ce que sur le mode

10. Cela comprend toutes les langues orales, mais également les langues signées, longtemps stigmatisées comme n'étant pas de "vraies » langues. Voir Laura Ann Petito, "Are signed languages "real" languages ? Evidence from American Sign Language and Langue des Signes Québécoise », Signpost. International Quarterly of the Sign Linguistics Association, vol. 7, n 3, 1994, p. 173-182. 
évaluatif de la norme. L'école d'ailleurs prend en général la responsabilité de la mise en forme de cette compétence à travers l'enseignement de la langue maternelle et des langues étrangères. Autrement dit, le langage est objet de discours de toutes sortes, discours tenus par tout le monde dans des contextes très divers ${ }^{11}$.

Constamment en mouvance, ces représentations se modifient selon l'importance croissante ou décroissante d'une communauté linguistique à un moment et dans un lieu donnés. Elles sont donc tributaires du prestige que l'on confère aux langues et aux locuteurs de ces langues ${ }^{12}$, de la reconnaissance ou de la légitimité sociopolitique, juridique et symbolique dont ces langues et ceux qui les parlent se prévalent. Certaines langues sont ainsi perçues comme plus belles, plus éloquentes et plus en mesure de traiter de certains domaines (par exemple, les sciences, les arts, l'amour), alors que d'autres sont jugées plus dures, moins aptes à la communication ou simplement trop vieilles. Il en va de même des jugements portés sur les variétés dialectales de certaines langues, hâtivement qualifiées de demi-langues, de langues vulgaires, bref de langues inaptes à la communication et à la compréhension ${ }^{13}$.

Les images et les qualificatifs transmis par les discours sur des langues ou des variétés de langue agissent sur les attitudes que 1'on adopte à leur égard, et ces attitudes « ont des retombées sur le comportement linguistique ${ }^{14} »$, puisqu'elles sont intériorisées dans les comportements langagiers et dans les choix sociolinguistiques : telle langue ou telle variété dialectale est ainsi privilégiée au détriment d'une autre, telle langue ou telle variété dialectale est apprise plutôt qu'une autre.

11. Bernard Py, « Pour une approche linguistique des représentations sociales », Langages, vol. 38, $\mathrm{n}^{\circ}$ 154, 2004, p. 7.

12. Le prestige attribué aux langues est étroitement lié au poids politique et économique dont disposent les locuteurs de ces langues.

13. Voir Marina Yaguello, Catalogue des idées reçues sur la langue, Paris, Seuil, 1988, 157 p.

14. Louis-Jean Calvet, cité dans Thierry Bulot, "Variations et normes d'une langue », dans Thierry Bulot et Philippe Blanchet (dir.), Dynamiques de la langue française au $21^{\text {ième }}$ siècle : une introduction à la sociolinguistique, CREA/CIM, Université de Rennes 2, 2011, www.sociolinguistique.fr (consulté le 9 mars 2018). 
La représentation linguistique ou « l'image mentale que les locuteurs se font de leur langue, de leur façon de la parler, de sa légitimité ${ }^{15}$ » et les attitudes linguistiques - les jugements qui en découlent, et qui valorisent ou dévalorisent la façon de parler - ont en commun le trait épilinguistique du fait qu'elles sont transmises dans le discours : «c'est par le discours [que les représentations sociales] existent et se diffusent dans le tissu social $^{16} »$. Or, les interprétations qui circulent sur les langues sont souvent empreintes d'une méconnaissance de la complexité langagière et de l'évolution des langues où l'on « ne fait pas toujours la part de 1'opinion et du savoir ${ }^{17} »$, entre autres sur le phénomène de la variation linguistique, sur les registres de langue, sur les différentes normes implicites et explicites dans l'usage d'une langue, sur l'évolution différentielle de l'oral et de l'écrit. Les représentations sont donc souvent le fruit d'idées reçues (croyances collectives ou stéréotypées) et les jugements qui en sont les corollaires tendent à mener à la dévalorisation de phénomènes qui, en d'autres lieux et à d'autres moments, auraient pu être jugés tout à fait naturels.

L'extrême flexibilité qui caractérise les langues n'est pas toujours bien perçue par les locuteurs, qui ont souvent tendance à concevoir leur langue comme une entité « une et indivisible ». [...] [L]e recours à des étiquettes comme le français, le japonais ou l'italien $[\ldots]$ cache le caractère variable et hétérogène des langues et ne rendent pas bien compte de l'existence de plusieurs variétés de langue différentes. Généralement, les variétés sont jugées en fonction du prestige des locuteurs qui s'en servent : la langue populaire est stigmatisée, la langue de l'élite est valorisée ${ }^{18}$.

15. Matthieu LeBlanc, « Le Français, langue minoritaire, en milieu de travail : des représentations linguistiques à l'insécurité linguistique ", Nouvelles perspectives en sciences sociales. Revue internationale de systémique complexe et d'études relationnelles, vol. 6, n 1, 2010, p. 19.

16. Bernard Py, « Pour une approche linguistique des représentations sociales », op. cit., p. 6.

17. Marty Laforest l'illustre bien dans son ouvrage États d'âme, états de langue. Essai sur le français parlé au Québec, 2e édition, Québec, Nuit Blanche Éditeur, 2007, p. 10.

18. Wim Remysen, « Le Français et la variation linguistique », dans Hélène 
On confère d'ailleurs souvent à ces images généralisées et tenues comme commune mesure une apparente véracité du fait que, de par leur diffusion, elles se présentent comme des « constats factuels avérés ${ }^{19}$ ». C'est ainsi que les représentations «sont relativement viables, même et surtout en l'absence d'argumentation $^{20} »$. À titre d'exemple, plusieurs personnes soutiennent, de nos jours, que l'anglais est plus concis et plus apte à forger de nouveaux mots : ce sont des velléités communes entretenues pour justifier son usage, comme si les autres langues étaient inaptes à la concision, étaient incapables d'innovation.

Parler des représentations, c'est parler de l'attitude qu'ont les locuteurs à l'égard de leur idiome et de la légitimité symbolique qu'ils lui confèrent. Ce faisant, cela permet de comprendre les représentations qui circulent au sujet d'une langue, de cerner la force symbolique que celle-ci revêt et de saisir les schèmes de pensée qui illustrent et qui expliquent l'image que l'on s'en fait ${ }^{21}$. Cette compréhension de «[...] la dynamique des langues et des variétés d'une même langue parlées sur un territoire donné, qui est toujours complexe $[\ldots]$ constitue un préalable nécessaire à toute opération d'aménagement linguistique ${ }^{22} »$, opération qui cherche à

Cajolet-Laganière, Pierre Martel et Chantal-Édith Masson (dir.), Usito, dictionnaire général de la langue française, 2013, https://www.usito.com/dictio (consulté le 25 août 2017). op. cit.

19. Bernard Py, «Pour une approche linguistique des représentations sociales »,

20. Ibid., p. 8 .

21. Pour l'apport des représentations sociales dans le traitement des discours, voir Julie Boissonneault, « Divergences et convergences dans les représentations du bilinguisme ", Francophonies d'Amérique, n 25, 2008, p. 19-45. Voir aussi Jean Claude Abric (dir.), Pratiques sociales et représentations, Paris, Presses universitaires de France, 1994 ; Willem Doise et al., Représentations sociales et analyses de données, Grenoble, Presses universitaires de Grenoble, 1992, 261 p. ; Christian Guimelli, «Étude expérimentale de la représentation sociale comme guide pour l'action : effets de l'implication et de la perception de la situation ", dans Monique Lebrun (dir.), Les Représentations sociales. Des méthodes de recherche aux problèmes de société, Outremont (Québec), Les Éditions Logiques, 2001, p. 93-108.

22. Marty Laforest, "Attitudes, préjugés et opinions sur la langue ", dans Claude Verreault, Louis Mercier et Thomas Lavoie (dir.), Le Français, une langue à apprivoiser. Textes des conférences prononcées au Musée de la civilisation (Québec, 2000-2001) dans le cadre de l'exposition Une grande langue : le français dans tous ses états, Québec, Presses de l'Université Laval, 2002, p. 86. 
renverser l'image dépréciative et l'usage dévalorisé d'une langue. Pour réussir, toute opération d'aménagement linguistique se doit ainsi d'agir à la fois sur le code (le matériau linguistique en soi) et sur le statut de ce code (son usage et sa reconnaissance dans la sphère publique).

Les sociolinguistes ont été nombreux à s'intéresser au phénomène des représentations linguistiques et des attitudes à l'égard du français, notamment en Europe (France, Belgique et Suisse), en Afrique et au Canada (particulièrement au Québec et au NouveauBrunswick), cherchant à expliquer la dialectique entre le français de référence (la langue normée) et la langue vernaculaire (les français régionaux). Le phénomène de l'autodépréciation est récurrent dans ces études.

[L]es locuteurs qui utilisent une variété de langue peu prestigieuse en ont une image négative, souvent plus négative encore que l'image qu'en ont les locuteurs s'exprimant dans la variété valorisée. Cette autodépréciation est constitutive d'un sentiment d'insécurité linguistique ${ }^{23}$.

\subsection{La variation linguistique : un phénomène universel}

Dans son article, Koscielniak aborde la question de l'insécurité linguistique des Franco-Ontariens en faisant valoir qu'elle est étroitement liée à la représentation qu'ils se font du français qu'ils parlent et des attitudes qu'ils manifestent quant à l'usage d'une langue familière. Afin de mettre les pendules à l'heure quant à certains usages courants en français parlé qui se démarquent du français de référence, elle explique les «spécificités francoontariennes » par l'évolution des langues, par les différences intergénérationnelles, par les registres que sont le familier et le soutenu et par l'emprunt à la langue anglaise.

Tous ces phénomènes, universels dans les langues vivantes ${ }^{24}$, sont le reflet de l'innovation qui s'opère dans une langue et de la variation qui en découle, et ce, plus rapidement à l'oral qu'à

23. Ibid., p. 88.

24. Les langues vivantes et « bien portantes » tendent à la variation lorsqu'elles sont parlées par plusieurs locuteurs. Seules les langues moribondes se fossilisent et cessent de varier. 
l'écrit. En s'exprimant, une personne tend vers la régularisation (le besoin d'uniformiser, de généraliser, d'éliminer ce qui fait exception), d'où l'apparition de variantes non conformes aux normes explicites ; elle tend vers la brièveté (le besoin d'abréger, de dire de façon plus économe), d'où les élisions, les fusions, les troncations, les omissions ; elle tend aussi vers l'expressivité 25 , d'où l'emprunt à d'autres langues ou à d'autres registres d'une langue donnée, et la création de mots, d'expressions. Ces besoins font appel aux processus d'innovation, dont l'emprunt ${ }^{26}$, entraînant de nouveaux vocables ou de nouvelles acceptions aux aires sémantiques : il en émane ainsi des variantes pour exprimer une même chose, variantes qui participent au renouvellement d'une langue sans forcément mettre en péril le fonds commun que partagent les locuteurs de cette langue ou lui nuire. Ces variantes s'inscrivent dans l'espace temporel, y compris entre les générations, dans l'espace géographique, dans les groupes socioéconomiques ou d'appartenance socioprofessionnelle, dans le style propre à chaque individu et le contexte situationnel, ainsi que dans l'usage de la langue à l'oral et à l'écrit ${ }^{27}$. « Le français est [ainsi], comme toutes les langues vivantes, soumis à des forces de diversification qui font en sorte que ses locuteurs ne parlent pas tous exactement de la même façon ${ }^{28}$. »

25. Voir Michèle Perret, Introduction àl'histoire de la langue française, $4^{e}$ édition, [s.1.], Armand Colin, 2014, p. 107-111.

26. Au processus d'innovation qu'est l'emprunt, s'ajoutent la dérivation (par préfixation et suffixation), l'extension de sens, la troncation (et la siglaison) et la composition (y compris les mots-valises).

27. Les linguistes réfèrent à ces différentes formes de variation par les termes « variation diachronique (ou intergénérationnelle) » pour parler de l'espace temporel, « variation diatopique » pour référer aux différences de milieux géographiques, "variation diastratique " pour souligner les distinctions entre les groupes, "variation diaphasique (ou situationnelle) » lorsqu'il est question de l'idiolecte, et « variation diamnésique " pour distinguer les formes relevant de l'oral de celles relevant de l'écrit. Voir Françoise Gadet, « La Variation : le français dans l'espace social, régional et international ", dans Marina Yaguello (dir.), Le Grand livre de la langue française, Paris, Éditions du Seuil, p. 91-152 ; et Julie Boissonneault, « Rétrospective sur le français parlé en Ontario ", Revue du Nouvel-Ontario, $\mathrm{n}^{\circ} 41,2016$, p. 200-202.

28. Wim Remysen, « Le Français et la variation linguistique », op. cit. 
Il va donc de soi que l'on ne parle pas tout à fait de la même manière d'une génération à l'autre, selon que l'on habite en un lieu ou en un autre, selon que l'on exerce telle profession ou tel métier, selon que l'on ait tel ou tel niveau d'instruction, selon que l'on communique dans un contexte familier et intime plutôt que dans un contexte formel et public, selon que l'on s'exprime spontanément à l'oral ou que l'on communique à l'écrit. Ces variantes vont toucher tous les aspects de la langue, y compris les tournures de phrases et la prononciation, bien qu'elles soient plus présentes dans le lexique.

Les linguistes qui se sont intéressés à l'expression orale en Ontario français ont relevé plusieurs de ces variantes, les expliquant soit par le maintien de formes et de vocables qui ne sont plus d'usage hors de l'Amérique (par exemple, je vas-je vais ${ }^{29}$ ou abrier-couvrir $)^{30}$, soit par l'extension de l'aire sémantique d'un mot (comme c'est le cas du mot « tour» dans la locution donner un tour à quelqu'un ${ }^{31}$ ). Ils se sont aussi penchés sur les usages parallèles qui marquent les registres ${ }^{32}$, comme l'alternance entre l'auxiliaire avoir et être, la disparition du subjonctif ${ }^{33}$ et l'usage

29. Raymond Mougeon, Terry Nadasdi et Katherine Rehner, «Évolution de l'alternance je vas / je vais / je m'en vas / je m'en vais / $m$ 'as dans le parler d'adolescents franco-ontariens (1978-2005) », dans Luc Baronian et France Martineau (dir.), Le Français d'un continent à l'autre. Mélanges offerts à Charles Morin, Québec, Presses de l'Université Laval, 2009, p. 327-373.

30. Voir, tout particulièrement, l'ouvrage de Marcel Bénéteau et Peter W. Halford (Mots choisis, Trois cents ans de francophonie au Détroit du lac Érié, Ottawa, Les Presses de 1'Université d'Ottawa, 2008, 532 p.), qui recèle des usages propres au Sud-Ouest, l'une des plus vieilles régions de colonisation française en Ontario. Voir aussi Alain Thomas, "Le Franco-ontarien : portrait linguistique », dans Raymond Mougeon et Édouard Beniak (dir.), Le Français canadien parlé hors Québec. Aperçu sociolinguistique, Québec, Presses de l’Université Laval, 1989, p. 19-35.

31. Julie Boissonneault, « Rétrospective sur le français parlé en Ontario »,op. cit., p. 216 ; Julie Boissonneault et Ali Reguigui, « Au-delà des technolectes. Problématique de traitement du corpus du français parlé en Ontario », dans Leïla Messaoudi et Pierre Lerat (dir.), Les Technolectes / Langues spécialisées en contexte plurilingue, Rabat (Maroc), Publication du laboratoire Langage et société CNRST-Urac56 de l'Université Ibn Tofaïl, en collaboration avec le Réseau maghrébin des technolectes (REMATE), 2014, p. 429-444.

32. Voir, entre autres, Terry Nadasdi, Raymond Mougeon et Katherine Rehner, "Expression de la notion de "véhicule automobile" dans le parler des adolescents francophones de l'Ontario ", Francophonies d'Amérique, no 17, 2004, p. 91-106.

33. Shana Poplack et Stephen Levey, «Variabilité et changement dans les 
concurrent de locutions telles que (ça) fait que / donc / alors et so selon la classe sociale et l'âge des locuteurs ${ }^{34}$, notant souvent une certaine dévernacularisation, c'est-à-dire la perte de la forme familière chez des locuteurs ${ }^{35}$. Ils ont cherché à distinguer les innovations qui relevaient de l'interférence de l'anglais de celles que l'on pouvait attribuer à des changements intrasystémiques, illustrant à maintes reprises que les variantes dans le discours ne peuvent pas systématiquement être attribuées au simple contact de l'anglais ${ }^{36}$.

Or, bien que plusieurs de ces variantes soient attestées, expliquées et contextualisées, elles semblent toujours faire 1'objet - dans les discours populaires tenus sur la langue francoontarienne - de jugements sans discernement. Il s'en dégage alors l'idée d'un usage reçu et interprété comme l'attestation d'un mauvais parler. Toute idée fausse ou partielle, érigée en généralisation, peut ainsi devenir une idée commune et nourrir un discours peu reluisant, qui, par ricochet, entretient une image communément dépréciative de la variété de langue parlée et des locuteurs eux-mêmes.

\section{DU COMPLEXE À TENIR UN DISCOURS EN « BON 》 FRANÇAIS}

2.1. La compétence linguistique : entre la sécurité et l'insécurité À l'époque où il était chroniqueur à CBON (Radio-Canada,

grammaires en contact », dans France Martineau et Terry Nadasdi (dir.), Le Français en contact. Hommage à Raymond Mougeon, Québec, Presses de l'Université Laval, 2011, p. 247-280.

34. Davy Bigot, « Identité et variation linguistique : les données de Casselman (Ontario) », Revue du Nouvel-Ontario, $\mathrm{n}^{\circ} 41,2016$, p. 216. Les travaux de Bigot s'inscrivent dans le prolongement des travaux menés par Raymond Mougeon à Hawkesbury.

35. Raymond Mougeon et Terry Nadasdi, « Discontinuités variationnelles dans le parler des adolescents franco-ontariens », Revue du Nouvel-Ontario, n 20,1996 , p. 51-76. Cette perte de la forme vernaculaire (familière) se produit essentiellement chez les locuteurs qui n'acquièrent pas la langue au foyer, mais l'apprennent dans un cadre scolaire.

36. Voir Raymond Mougeon, « La Question de l'interférence de l'anglais à la lumière de la sociolinguistique ", dans Claude Poirier et al. (dir.), Langue, espace, société. Les variétés du français en Amérique du Nord, Québec, Presses de l'Université Laval, 1994, p. 25-40. 
Sudbury), Normand Renaud relate qu' « [a]u moins la moitié des personnes à qui je demand[ais] une entrevue me dis[ai]ent d'abord ["Mon français est pas très bon"]. Au moins cent pour cent [sic] d'entre elles me dis[ai]ent ensuite plein de choses bien assez bonnes pour passer à la radio ${ }^{37} \gg$.

Cette perception d'aise ou de malaise à s'exprimer est le phénomène de la sécurité (dont on parle rarement) ou de l'insécurité linguistique, respectivement. Se percevoir compétent ou se percevoir incompétent dans une langue donnée n'est pas toujours un corollaire de la maîtrise qu'a un individu à s'exprimer dans cette langue, comme l'a noté Renaud. C'est le cas, cependant, lorsqu' une personne est en instance d'apprentissage d'une langue : sentir un malaise à s'exprimer est alors chose tout à fait naturelle.

Là où le bât blesse, c'est lorsqu'il s'agit, aux yeux d'un locuteur, d'une incompétence qu'il attribue à l'usage qu'il fait de sa langue première ou d'une langue à laquelle ou par laquelle il s'identifie, en fonction d'un discours sur la pratique jugée selon qu'elle soit conforme ou non aux attentes prescrites ${ }^{38}$. Le jugement résulte alors généralement de représentations linguistiques, c'està-dire d'un construit érigé sur les diktats que véhicule la société à l'égard de la langue, ou, plus particulièrement, à l'égard de la variété de langue qu'il utilise.

[L']insécurité linguistique [est] la prise de conscience, par les locuteurs, d'une distance entre leur idiolecte (ou leur sociolecte) et une langue qu'ils reconnaissent comme légitime parce qu'elle est celle de la classe dominante, ou celle d'autres communautés où l'on parle un français "pur", non abâtardi par les interférences avec un autre idiome, ou encore celle de locuteurs fictifs détenteurs de LA norme véhiculée par l'institution scolaire ${ }^{39}$.

37. Normand Renaud, «Le Bon Français, celui que nous parlons », De face et de billet. Une chronique d'humeur franco-ontarienne, Sudbury, Prise de parole, 2002, p. 175.

38. Marie-Louise Moreau, "La Pluralité des normes dans la francophonie », DiversCité Langues, vol. IV, 1999, http://www.teluq.uquebec.ca/diverscite (consulté le 9 mars 2018).

39. Michel Francard, L'Insécurité linguistique en Communauté française de Belgique, Bruxelles, Ministère de la Culture, Service de la Langue française, 1993, p. 13. 
Souffrir d'insécurité linguistique ne signifie donc pas de façon sine qua non que cette personne soit incompétente. Il se peut qu'elle ne se sente pas à la hauteur d'autres locuteurs ou du même calibre qu'eux, en raison d'une perception d'une compétence moindre ou pressentie comme telle, soit dans une variété dialectale d'une langue particulière, soit dans un registre de cette langue.

L'insécurité linguistique, en tant que manifestation d'un complexe d'infériorité à s'exprimer dans une langue donnée, n'est pas le propre du parler des Franco-Ontariens. La question a fait l'objet d'études auprès de nombreuses communautés linguistiques de langue française ${ }^{40}$, dont les francophonies québécoise et acadienne. Cela s'explique par les travaux d'aménagement de la langue française dans ces deux provinces, travaux de portée sociopolitique menés en parallèle à l'aménagement du français comme langue officielle ou co-officielle. Peu nombreux, cependant, sont les chercheurs qui se sont penchés sur les représentations et les attitudes qui alimentent l'insécurité linguistique en Ontario français ${ }^{41}$, la langue n'y ayant pas fait l'objet d'une description critique aussi concertée et son usage ne bénéficiant pas de la même reconnaissance sur la scène publique. Or, les travaux d'aménagement linguistique ayant comme visée d'agir sur la légitimité sociopolitique et juridique d'une langue agissent,

40. Dans une enquête sur la perception qu'entretiennent des locuteurs provençaux de leur parler à l'égard du français parisien, Lawrence Kuiper constate ce qui suit : " They seem convinced that, despite all their efforts, their own speech is in some way inferior, and when asked to perform in the target variety, they fail to perceive that they have been doing so all along. The major difference between their native speech and that of Paris exists in their minds, the product of their having adopted not only the Parisian norms for correctness, but the Parisian evaluation of their linguistic performance (Kuiper, 2005, p. 47) » cité dans Sylvie Roy, "Qui décide du meilleur français? Représentations des variétés linguistiques du français en immersion », The Canadian Journal of Applied Linguistics / Revue canadienne de linguistique appliquée, vol. 15, n 1, 2012, p. 3-4.

41. Roger Lozon, « Les Jeunes du sud-ouest ontarien : représentations et sentiments linguistiques », Francophonies d'Amérique, n 12, 2001, p 83-92 ; Tina Desabrais, «“C'est pas pour moi”. L'influence de l'insécurité linguistique sur le choix de rédiger une thèse ou pas : le cas d'Angèle ", Liaison. La Revue des arts, hiver 2013, n' 162, p. 8-9 ; Sylvie Roy, « Le Bilinguisme, les jeunes et le milieu du travail : maintien d'une communauté ? ", Francophonies d'Amérique, no 12, 2001, p. 51-59. 
par ricochet, sur sa valorisation - sa légitimité symbolique - aux yeux des locuteurs.

\subsection{Contrer l'insécurité linguistique pour légitimer le français au Québec et en Acadie}

La question des représentations et des attitudes à l'égard du français, tant dans l'optique de la qualité de la langue que dans celle de son statut a été, et continue d'être, au cœur des préoccupations des Québécois ${ }^{42}$, préoccupations qualifiées de «véritable sport national $^{43} »$, tant sont nombreux les essais, les traités, les lettres d'opinion et les ouvrages qui s'y sont consacrés. Il n'est dès lors pas surprenant que la qualité et le statut de la langue française aient été promus - en particulier dans la Charte de la langue française-comme les « deux principaux piliers de la législation québécoise ${ }^{44} \gg$ en matière d'aménagement linguistique.

Dans son ouvrage Méchante langue. La légitimité linguistique du français parlé au Québec, Chantal Bouchard lève le voile sur la montée de l'insécurité linguistique par l'étude de témoignages, de métadiscours (traités et remarques sur la langue) et de la littérature qui ont marqué l'évolution de la variation du français parlé au Québec en parallèle avec celle du statut socio-politicoéconomique des Canadiens français ${ }^{45}$. Elle montre comment le phénomène de l'insécurité linguistique chez les Québécois s'est infiltré dans le discours collectif au milieu du XIX ${ }^{\mathrm{e}}$ siècle en faisant état de descriptions et d'observations impressionnistes sur le français parlé des Canadiens français qui ont alimenté, pendant

42. Hélène Cajolet-Laganière et Pierre Martel,La Qualitéde la langue au Québec, Québec, Institut québécois de recherche sur la culture, collection " Diagnostic », $\mathrm{n}^{\circ} 18,1995,167 \mathrm{p}$.

43. Marty Laforest, États d'âme, états de langue..., op. cit., p. 9.

44. Elke Laur, «La Qualité, le statut et la perception du français au Québec », Revue d'aménagement linguistique, numéro hors-série, automne 2002, p. 147.

45. Bouchard attribue l'évolution du français en France et en Amérique à des événements sociopolitiques différents. La première prise de conscience des Canadiens français quant à l'évolution de leur langue se serait produite en 1841-1842. Voir Chantal Bouchard, Méchante langue. La légitimité linguistique du français parlé au Québec, Montréal, Presses de l’Université de Montréal, 2011, 171 p. 
près d'un siècle, des représentations dépréciatives au sujet de la langue, au point de miner la légitimité même de la langue française en Amérique.

D'autres chercheurs ont développé des arguments visant à contrer les représentations et les attitudes qui sous-tendaient l'insécurité linguistique collective face au français parlé au Québec. Marty Laforest déconstruit ainsi, dans États d'âme, états de langue. Essai sur le français parlé au Québec ${ }^{46}$, les idées reçues sur la langue parlée par les Québécois et présentées comme des vérités sans faille. Elle reproche aux tenants de ces discours d'arguer que la langue familière ou populaire - le parler quotidien est la seule expression dont sont capables les Québécois et elle en fait la démonstration. Dans son ouvrage La Langue rapaillée. Combattre l'insécurité linguistique des Québécois ${ }^{47}$, Anne-Marie Beaudoin-Bégin est tout aussi explicite.

À partir des années 1970, les Québécois ont agi sur cette insécurité linguistique « formelle » par l'aménagement du code (les grands travaux terminologiques de l'Office québécois de la langue française), ainsi que sur l'insécurité linguistique "statutaire » par l'aménagement du statut du français (son officialisation, son imposition comme langue de travail et sa plus grande visibilité publique, entre autres $)^{48}$. Dans l'un ou l'autre des cas, la langue a fait l'objet d'un travail exhaustif de description - observation des variantes, explication de leur provenance, attestation de leur présence, etc.--, ce qui a permis à la collectivité franco-québécoise de faire le point, en connaissance de cause, sur les traits et les usages linguistiques à maintenir selon les contextes et sur ceux

46. Marty Laforest, États d'âme, états de langue... op. cit.

47. Anne-Marie Beaudoin-Bégin, La Langue rapaillée. Combattre l'insécurité linguistique des Québécois, [s.1.], Somme toute, 2015, 114 p.

48. Nous empruntons cette distinction - sécurité-insécurité formelle ou statutaire - à Louis-Jean Calvet afin de différencier l'aménagement qui touche le code de celui qui touche le statut de ce code. Marie-Louise Moreau fait aussi cette distinction, mais parle d'insécurité linguistique « agie » lorsqu'elle se rapporte à une préoccupation excessive de la forme correcte et d'insécurité linguistique " dite » lorsqu'elle touche à l'aspect statutaire de la langue. Voir Thierry Bulot et Philippe Blanchet (dir.), Dynamiques de la langue française au $21^{i e ̀ m e}$ siècle..., op. cit. 
à élaguer. Seul un tel travail de description de la langue, de première importance pour contrer les représentations linguistiques qui circulent, permet la (re)valorisation et la légitimation ${ }^{49} \mathrm{~d}^{\prime} u n e$ langue, ce qui, en retour, réduit l'insécurité linguistique.

La question de l'insécurité linguistique a également interpellé les chercheurs de l'Acadie, qui ont, au fil des ans, travaillé à faire le point sur la trialectique que constituent l'acadien, le français de référence et le chiac dans le but d'agir sur les représentations.

Les nombreux travaux de description du français acadien ont fait valoir la richesse de sa spécificité et l'ont situé dans l'histoire, lui conférant, par le fait même, une légitimité historique. Les travaux de Louise Péronnet, de Lise Dubois et d'Annette Boudreau $^{50}$, parmi d'autres, ont beaucoup contribué à mieux comprendre ces trois variétés dialectales et la place qu'elles occupent dans l'expression des Acadiens, soulignant la richesse des parlers régionaux au patrimoine linguistique de la langue française. Le français acadien s'inscrit désormais comme l'une des deux grandes variétés de français parlé au Canada, à l'instar du français laurentien, «la variété anciennement appelée "francocanadienne" [parlée dans] la plus grande partie du Québec et [dans] toutes les aires francophones du Canada qui se trouvent à l'ouest de celui-ci ${ }^{51} »$.

49. Voir Louise Péronnet, « Place de la description dans la représentation d'une langue et dans la légitimité linguistique : l'exemple de l'Atlas linguistique du vocabulaire maritime acadien », Revue québécoise de linguistique, vol. 26, n 2, 1998, p. 69-80 ; Jean-Claude Corbeil, L'Aménagement linguistique du Québec, Montréal, Guérin, 1980, 153 p.

50. Voir, entre autres, Annette Boudreau, « La Construction des représentations linguistiques : le cas de l'Acadie ", Revue canadienne de linguistique, vol. 54, $\mathrm{n}^{\circ} 3$, 2009, p. 439-459; Annette Boudreau et Lise Dubois, « Français, Acadien, Acadjonne : Competing Discourses on Language Preservation Along the Shores of the Baie Sainte-Marie », dans Alexandre Duchêne et Monica Heller (dir.), Discourses of Endangerment: Interest and Ideology in the Defense of Languages, Londres, Continuum International, 2007, p. 99-121; Louise Péronnet, «Place de la description... », op. cit.

51. Claude Poirier, «L'Assibilation des occlusives /t/ et/d/ au Québec : le point sur la question », dans Luc Baronian et France Martineau (dir.), Le Français d'un continent à l'autre, op. cit., p. 385. Voir aussi Marie-Hélène Côté, " La Longueur vocalique devant consonne allongeante en contexte final et dérivé en français laurentien "), dans Carmen Leblanc, France Martineau et Yves Frenette (dir.), Vues sur les français d'ici, Québec, Presses de l’Université Laval, 2010, p. 50. 
Matthieu LeBlanc ${ }^{52}$, pour qui l'insécurité linguistique est associée invariablement aux représentations et aux situations de diglossie, s'est intéressé à la question auprès des fonctionnaires francophones du Nouveau-Brunswick travaillant en milieu bilingue anglais-français. Ses travaux établissent le lien étroit entre les manifestations d'insécurité linguistique et la dépréciation du parler, mettant en évidence l'écart entre l'autoévaluation dépréciative de la compétence en français et les compétences réelles observées, entre les attentes véhiculées par l'institution scolaire et le regard des autres.

Tant au Québec qu'en Acadie, le travail des chercheurs sur la description de la langue a été consigné dans des ouvrages de référence qui attestent de la variation et qui l'expliquent. Glossaires, dictionnaires, bulletins terminologiques sont ainsi à la disposition des locuteurs du français, leur permettant de comprendre ce qu'il en est réellement et de porter un jugement en connaissance de cause. Sans ouvrages de référence, il est difficile de véhiculer un discours qui contre les idées reçues.

\subsection{Les Franco-Ontariens face à la langue}

En Ontario, l'usage de la langue française a fait l'objet de nombreuses études - toutes disciplines confondues. Toutefois, la représentation de la langue et ses retombées sur le comportement langagier, bien que fréquemment soulignées en exergue par les chercheurs, ont peu fait l'objet d'études critiques et détaillées. Les nombreux travaux de Raymond Mougeon et collaborateurs ont permis de lever le voile sur la prémisse que tous les locuteurs du français en Ontario ne peuvent être étudiés comme un seul groupe du fait qu'ils n'ont pas tous le français comme langue première ou comme seule langue première et qu'ils n'en font pas le même usage dans leurs milieux. Ils ont ainsi proposé de distinguer les locuteurs du français en fonction de la fréquence de leur usage de la langue ${ }^{53}$.

52. Matthieu LeBlanc, « Le Français, langue minoritaire, en milieu de travail...", op. cit.

53. Mougeon et collaborateurs qualifient de « locuteur non restreint », l'individu 
Les quelques chercheurs qui se sont intéressés au phénomène de l'insécurité linguistique s'inscrivent dans des contextes particuliers et dans des régions spécifiques; c'est le cas, notamment, de Roger Lozon pour le Sud-Ouest ontarien et de Tina Desabrais pour le milieu universitaire de l'Est ${ }^{54}$.

Alors que Tina Desabrais fait la démonstration que le rapport à la langue - qu'il soit valorisant ou dévalorisant - agit sur la réussite ou sur l'échec d'étudiants aux études supérieures de l'Université d'Ottawa, l'enquête menée par Lozon se penche sur la représentation qu'entretiennent de jeunes locuteurs francophones habitant la région de Chatham-Kent à l'égard des variétés que sont le français régional (langue parlée) et le français de référence véhiculé par l'école. Il s'intéresse plus particulièrement à la perception de leurs compétences langagières en français et à leurs sentiments linguistiques selon les situations dans lesquelles ils s'expriment en français. Son analyse met de l'avant le constat que l'insécurité qu'ils ressentent à s'exprimer en français est nourrie par l'idée que leur langue n'est qu'un mélange de français et d'anglais et qu'elle n'a pas sa place dans l'espace public ${ }^{55}$. Ces représentations corroborent l'idée que le discours dépréciatif sur une langue et la légitimité de son usage nourrissent le sentiment d'incompétence, que celui-ci soit réel ou non.

L'analyse du discours des jeunes dans le Sud-Ouest ontarien est très révélateur $[\mathrm{sic}]$ de leurs représentations et de leurs sentiments linguistiques. Les jeunes nous indiquent d'abord qu'ils ont très peu d'espaces où ils ont l'occasion d'utiliser et de perfectionner leur français [...]. D'autres ne se sentent pas

pour qui le français est la langue dominante et d'usage majoritaire, de « locuteur semi-restreint » celui qui utilise autant le français que l'anglais dans ses interactions quotidiennes, et de " locuteur restreint » celui qui s'exprime essentiellement en anglais.

54. Il s'agissait, dans les deux cas, de thèses doctorales. Voir Roger J. Lozon, "Représentations et sentiments linguistiques dans le sud-ouest ontarien ", thèse doctorale, Toronto, University of Toronto, 2004 ; Tina Desabrais, « Les Mots pour le dire... L'influence de l'(in)sécurité sur l'expérience d'étudiantes de milieux francophones minoritaires canadiens inscrites aux études supérieures à l'Université d'Ottawa », thèse doctorale, Ottawa, Université d'Ottawa, 2013, 359 p.

55. Roger Lozon, « Les Jeunes du sud-ouest ontarien... », op. cit. 
assez à l'aise avec la variété de français qu'ils possèdent pour l'utiliser en public. Le fait de se représenter le français régional comme un français qui est un mélange de français et d'anglais et de s'interroger parfois sur sa légitimité nuit également à l'utilisation de cette langue dans les espaces sociaux qu'occupent les jeunes, et ce, même lorsqu'ils se retrouvent parmi d'autres personnes qui connaissent le français ${ }^{56}$.

Un rapport paru en 2014, et donnant suite à une consultation auprès de la jeunesse franco-ontarienne de toutes les régions de la province ${ }^{57}$, confirme les sentiments de sécurité et d'insécurité linguistique qu'entretiennent des jeunes franco-ontariens à l'égard de leur parole. Aux dires des jeunes franco-ontariens, leurs sentiments sont largement tributaires de jugements - positifs et négatifs - portés sur la qualité de leur langue, tels que d'avoir un bel accent ou, à l'inverse, un mauvais accent ou un accent « bizarre ». La plupart des jeunes disent se sentir à l'aise de s'exprimer en français au sein de leur famille ou avec les amis francophones, voire en contexte scolaire, mais beaucoup moins à l'aise de le faire hors de ces milieux. Ils allèguent aussi que leur sentiment de malaise et d'insécurité augmente en présence de locuteurs non francophones et de locuteurs franco-québécois, en raison des commentaires qu'ils leur font.

\section{De la lÉGitimation et de LA RECONNAisSANCE DU FRANCO-ONTARIEN}

À la question de savoir qui décide de la légitimité d'une langue et, par le fait même, de la validité de l'expression de locuteurs dans une langue ou dans une variété de langue donnée, force est de répondre que c'est à la fois le locuteur individuel et membre d'une communauté linguistique, et la communauté en général. Le concept de légitimité linguistique, nous l'avons vu, se décline de deux façons. D’abord la légitimité socio-politico-juridique,

56. Ibid., p. 91.

57. Fédération de la jeunesse franco-ontarienne (FeSFo), Pour s'exprimer dans notre langue. Consultation sur l'insécurité linguistique et la jeunesse francoontarienne. Rapport final, novembre 2014, 16 p. 
celle qui touche le statut de la langue (légitimité statutaire) et qui lui donne droit de cité, puis la légitimité linguistique que nous avons qualifiée de symbolique, qui est propre à l'idiome et qui « relève de la conscience collective qu'une communauté développe envers sa propre langue ${ }^{58} \gg$. Une langue ou une variété de langue légitime se forme ainsi à la fois par une reconnaissance formelle (avec la mise en place d'organismes qui en font usage) et par une reconnaissance des différences ou des spécificités linguistiques qui constituent son usage (telles que véhiculées dans les ouvrages de référence et littéraires). Toute langue « légitime » est donc tributaire d'un aménagement positif. Le français parlé au Québec - le québécois - et le français parlé en Acadie l'acadien - ont été légitimés par une reconnaissance statutaire et linguistique ainsi que par la reconnaissance de leur spécificité et de leur raison d'être. Il ne s'agissait pas, dans l'un ou l'autre des cas, de placer ces variétés de français au-delà de toute autre, mais bien de reconnaître leur existence, leur place, leur raison d'être et d'avaliser leurs spécificités. C'est ce qui a contribué à réduire (sans les enrayer complètement) les sentiments d'insécurité linguistique des Franco-Québécois ou des Acadiens quant aux " couleurs » de leur parler et face à d'autres variétés de français. Peut-on en dire autant du français parlé en Ontario?

\subsection{La description du corpus de la langue}

Le locuteur du français parlé en Ontario ne dispose pas réellement d'ouvrages de référence attestés et aisément accessibles (glossaires, dictionnaires et bulletins terminologiques) qui font état d'un usage standardisé en Ontario tout en tenant compte des variantes qu'on y retrouve et qui en expliquent 1'usage ${ }^{59}$. Il doit alors se tourner vers des ouvrages qui tiennent compte du français

58. Louise Péronnet, «Place de la description... », op. cit., p. 70.

59. Il dispose toutefois d'une littérature, qui est à l'étude dans le système scolaire de langue française et qui est enseignée à l'universitaire. Bien que je ne tienne pas compte de cet apport important à l'aménagement d'une langue, c'est, je le rappelle, par l'entremise d'une romancière franco-ontarienne - Hélène Koscielniak - que la question de la légitimité a été remise sur la table en 2016. 
québécois ${ }^{60}$ ou de provenance française. Bien que les linguistes se soient affairés à décrire plusieurs spécificités du français parlé en Ontario depuis les années 1970-1980, leurs nombreux travaux - publiés dans les milieux universitaires - ne semblent pas avoir percé les discours tenus sur la langue. Il n'est donc pas étonnant que les idées dépréciatives sur la langue soient monnaie courante dans les représentations que les locuteurs s'en font.

Dans son texte, Koscielniak propose la création d'un dictionnaire et d'un organe de normalisation qui feraient état de la spécificité du français parlé en Ontario. Cette proposition, qu'elle qualifie de « folle audace » et de « farfelue », est loin de l'être lorsque nous comprenons que ces éléments contribuent à l'aménagement positif d'une langue, à sa légitimité statutaire et linguistique. Sa proposition est d'autant moins « folle » et « farfelue » qu'elle avait déjà fait l'objet d'une requête, en 1982, par un groupe de chercheurs, membres de l'Institut franco-ontarien de 1'Université Laurentienne de Sudbury, qui demandait la création d'un organisme provincial pour étudier et documenter la langue française en Ontario. Le Rapport pour la mise sur pied de l'Office de la langue française en Ontario était un projet collectif qui a vu le jour à la suite de la tenue de journées d'étude les 20 et 21 mai 1981 à la Laurentienne ${ }^{61}$. Le rapport proposait d'agir à la fois sur le statut de la langue française en Ontario et sur le code en normalisant la terminologie, en menant des enquêtes sociolinguistiques, en contribuant aux travaux terminologiques menés par le Bureau de traduction provincial, en faisant la promotion de la

60. Les travaux d'aménagement de la langue française au Québec ont mené à des ouvrages de référence qui la légitiment, dont le Multidictionnaire de la langue française de Marie-Éva de Villers, Le Dictionnaire québécois d'aujourd'hui (sous la direction de Jean-Claude Boulanger), le Trésor de la langue française au Québec (www.tlfq.ulaval.ca) et le Grand dictionnaire terminologique de l'Office québécois de la langue française (www.oqlf.gouv.qc.ca).

61. Rapport pour la mise sur pied de l'Office de la langue française en Ontario (Raison d'être, sommaire des journées d'étude des 20 et 21 mai 1982, étude de faisabilité), Sudbury, Institut franco-ontarien, juin 1982, 52 p. L'évènement a été appuyé par un octroi du Secrétariat d'État. L'avant-propos est signé par Benoît Cazabon ; le rapport est rédigé par Michel Laurier ; les membres du comité organisateur des journées d'étude sont Georges Bélanger, Gaétan Gervais, Paul Jinot et Jacques Roy avec la collaboration de Serge Dignard. 
langue française et en offrant des services linguistiques spécialisés, notamment en matière d'apprentissage et d'évaluation de la compétence en français langue seconde.

L'usage de plus en plus répandu du français entraîne des complications techniques. Saurons-nous nous doter d'instruments pour que le français devienne un moyen d'épanouissement, de dépassement, de libération? ${ }^{62}$

Il s'agissait ainsi d'une requête de normalisation linguistique (par des travaux sur la spécificité du français ontarien) et de normalisation statutaire. La requête n'a pas eu de suite, mais, depuis, plusieurs des lacunes dont fait état le Rapport ont été comblées avec, entre autres choses, l'adoption de la Loi sur les services en français de 1986, la création du Commissariat aux services en français en 2007, puis du ministère des Affaires francophones en 2017. Il existe aussi de nombreux travaux et de nombreuses recherches qui documentent, qui expliquent et qui font état de l'usage du français en Ontario, mais moins nombreux sont ceux qui s'intéressent aux différentes façons dont la langue se décline, dans son expression en tant que telle. Dispersés dans le temps (de 1980 à aujourd'hui) et dans l'espace (auprès de populations spécifiques et dans des communautés majoritairement francophones - Hawkesbury, Casselman, Hearst - ou minoritaires - Sudbury, Toronto, Welland), ces travaux sont souvent reçus et interprétés comme l'attestation d'une langue en perte de vitesse, d'une langue hautement anglicisée ou d'un parler familier inapproprié pour que ne s'en dégage une compréhension valorisante du franco-ontarien.

Une description systématique et compréhensive du français parlé en Ontario est donc loin d'être faite, ce qui explique que les exemples qui en sont souvent donnés peuvent venir renforcer les idées reçues. Les exemples que relève Koscielniak de la langue parlée par les Franco-Ontariens l'illustrent bien : elle qualifie l'élision (comme dans J'ai de l'argent -J'ai d'l'argent et Es-tu allé à la partie ? - Es-tu allé à partie ?) et la fusion (je suis j'suis - chu) d'escamotage, alors que ces variantes témoignent

62. Ibid., p. i. 
du besoin de brièveté et font partie d'une expression familière. Il en va de même de la généralisation du pronom " on ", ou des généralisations verbales et temporelles (Faut que j'dors), de l'emprunt à l'anglais sous forme intégrée ou intégrale (bet pour pari, toffer pour endurer), des calques (Ça sonne mal, Ça fait du sens, Ça r'garde ben), et de certaines prononciations jugées " incorrectes » (frette, deboutte, faque). Toutes ces variantes s'expliquent, d'où l'importance de les décrire et de les expliquer, pour contrer l'idée communément admise que le français parlé en Ontario n'est que de l'anglais. Cette représentation, bien qu'invalidée par les linguistes depuis un certain temps, n'a pas rejoint le discours collectif ${ }^{63}$.

\subsection{La dénomination d'une langue : un acte délicat}

Koscielniak propose aussi de nommer le français parlé en Ontario par le vocable le « tarois ». Ce « tarois » correspond néanmoins, dans la définition qu'elle en donne et dans l'imaginaire collectif, à une forme familière hautement anglicisée de la langue. Qui plus est, elle le présente comme une autre langue lorsqu'elle réfère à des locuteurs trilingues s'exprimant en français, en anglais et en tarois. Le tarois est ainsi, selon elle, une langue orale, dérivée du français, qui, de par l'emprunt à son environnement, a mené à « un cocktail de français, d'anglais, de joual et d'autochtone, aromatisé d'une diversité d'accents ${ }^{64} »$. Il serait facile de dresser un parallèle entre l'imaginaire collectif à l'égard de ce " tarois » et celui à l'égard du chiac, d'une part, et d'entretenir l'idée que les francophones de l'Ontario n'ont que cette forme d'expression, ce seul registre, à leur portée, d'autre part.

Vouloir nommer la variété de langue, c'est vouloir reconnaître une différence, comme cela s'est fait au Québec et en Acadie. Cela permet de situer historiquement, géographiquement et linguistiquement ce qui la distingue des autres variétés constituant

63. Voir, entre autres, Raymond Mougeon, « La Question de l'interférence de l'anglais à la lumière de la sociolinguistique », op. cit. ; Alain Thomas, « Le Francoontarien : portrait linguistique », op. cit.

64. Hélène Koscielniak, « Le Tarois : dossier », op. cit., p. 7. 
le français. Les Franco-Québécois parlent québécois et non seulement joual ; ce faisant, ils parlent français. Les Acadiens parlent acadien et non seulement chiac ; ce faisant ils parlent français. Que dire des Franco-Ontariens? Ils ont en partage le français qu'ils parlent, tout comme le font les francophones québécois, acadiens, manitobains, belges, voire les Français. Les linguistes ont reconnu deux grandes variétés de français en sol canadien : le français laurentien et le français acadien. Suffit-il alors de dire que les Franco-Ontariens parlent le français laurentien ou le français canadien ? Ce serait là ignorer leur présence historique et géographique. Faut-il dire qu'ils s'expriment en " français parlé hors Québec », en « français de minoritaire »? Ces « deux [dernières] appellations [...] témoignent de la domination du point de vue québé[co]centrique ${ }^{65}$. Koscielniak propose « tarois », mais ce terme est affublé d'un sens dépréciatif. Nommer une variété de langue est un enjeu important et délicat, mais il peut aussi être une lame à double tranchant selon que l'on cherche à s'y identifier ou à s'en différencier, selon que l'on cherche à apprécier la variété ou à la déprécier.

Or, il existe un terme non dépréciatif pour parler de la langue française en Ontario - « le franco-ontarien ${ }^{66} »-$ qui situe la langue dans son contexte sociohistorique et sociopolitique. Les plus lointaines attestations que nous avons relevées de cette dénomination sont celles d'Alain Thomas (en 1989) et de Pierre Léon (en 1994), reprise par Yves-Charles Morin en 200267. Bien que le vocable soit

65. Monica Heller et Normand Labrie, «Langue, pouvoir et identité : une étude de cas, une approche théorique, une méthodologie », dans Monica Heller et Normand Labrie (dir.), Discours et identités. La francité canadienne entre modernité et mondialisation, [s.1.], Éditions modulaires européennes, 2003, p. 14.

66. Lors d'une communication à Newcastle (Royaume-Uni) en 2012, on nous a reproché l'usage de l'appellation « le franco-ontarien » pour parler du français parlé en Ontario, alléguant qu'il s'agissait de " français canadien », de " français laurentien ». Le reproche était porteur d'une dichotomie, puisque le français parlé au Québec, lui, se disait québécois. Voir Julie Boissonneault et Ali Reguigui, « Au-delà des technolectes...», op. cit.

67. Voir Alain Thomas, « Le Franco-ontarien : portrait linguistique », op. cit. ; Pierre Léon, "Recherches sur le phonétisme du franco-ontarien et sa mouvance ", dans Claude Poirier et al. (dir.), Langue, espace, société. Les variétés du français en Amérique du Nord, Presses de 1'Université Laval, 1994, p. 389, reprise par Yves- 
encore loin d'être d'usage commun et que d'aucuns lui préfèrent des termes plus généraux tels que « le français parlé en Ontario » ou « le français canadien », l'appellation « franco-ontarien » paraît dans des textes savants plus récents, notamment sous la plume de Robert Papen et Sandrine Hallion ${ }^{68}$, sous celles d'Albert Valdman, Julie Auger et Deborah Piston-Halten ${ }^{69}$ ainsi que sous la nôtre ${ }^{70}$.

\section{CONCLUSION}

Toute variété de langue devient légitime dès lors qu'elle acquiert un statut (légitimité statutaire) et que son expression, sa spécificité, est jugée apte à la communication (légitimité linguistique ou symbolique).

En Ontario, plusieurs pions pour cette légitimation sont en place sur l'échiquier. La langue française a une certaine reconnaissance statutaire, bien que son statut soit différencié puisqu'elle n'y est pas langue co-officielle. Elle est néanmoins langue de l'administration, langue de l'enseignement, langue des médias, langue de la culture, et ce, tant à l'écrit qu'à l'oral.

La deuxième forme de légitimité (dite formelle), celle qui touche davantage l'expression orale, la spécificité du code plutôt que le statut, dépend des représentations que l'on véhicule à son sujet. Or, pour comprendre les spécificités d'une variété de langue, comme c'est le cas du franco-ontarien, il faut l'aménager. Une grande part de cet aménagement relève de la primauté de la description que l'on en fait, description qui peut nourrir des idées reçues ou les contrer. Si la description est dépréciative, faussée ou incomplète, l'image qu'on s'en fera le sera tout autant; si la

Charles Morin, dans « Les Premiers Immigrants et la prononciation du français au Québec », Revue québécoise de linguistique, vol. 31, n 1, 2002, p. 44.

68. Robert A. Papen et Sandrine Hallion (dir.), À l'ouest des Grands Lacs : communautés francophones et variétés de français dans les Prairies et en ColombieBritannique, Québec, Presses de 1'Université Laval, 2014, 301 p.

69. Albert Valdman, Julie Auger et Deborah Piston-Halten (dir.), Le Français en Amérique du Nord. État présent, Québec, Presses de l'Université Laval, 2005, $583 \mathrm{p}$.

70. Voir la note 66. Julie Boissonneault et Ali Reguigui, « Au-delà des technolectes... », op. cit. 
description permet de comprendre les spécificités de la variété, de comprendre pourquoi elles se manifestent, de comprendre comment elles se distinguent ou non d'autres variétés de la même langue, de comprendre leur raison d'être, il est alors plus aisé de la légitimer dans le discours épilinguistique. Ce faisant, les locuteurs - en l'occurrence les Franco-Ontariens - pourraient se représenter leur langue parlée en connaissance de cause plutôt que d'adhérer à des lieux communs. Par ricochet, la représentation linguistique qu'ils se feraient de leur parler pourrait agir sur la compétence qu'ils croient détenir pour s'exprimer et sur leur comportement langagier.

Il importe donc de remettre les pendules à l'heure et de mieux comprendre les façons qu'ont les Franco-Ontariens de parler : comment ils innovent et ce qu'ils empruntent à l'anglais. Il importe aussi de comprendre comment l'oral se distingue de l'écrit, de faire le point sur le français de référence (le français dit standard ou neutre) par rapport aux multiples variétés dialectales de français ici et ailleurs, et de comprendre comment la variabilité n'est pas forcément le reflet d'une incompétence. Seule une telle compréhension, aménagée dans le discours, permettra de resituer les sentiments de sécurité et d'insécurité linguistiques, c'est-àdire d'aise ou de malaise à s'exprimer dans « un français vif, à l'usage encadré, et non écrasé par les normes prescriptives ${ }^{71} »$.

71. Marina Yaguello, Catalogue des idées reçues..., op. cit., p. 8. 